\title{
An Adaptive Synchronization Policy for Harvesting OAI-PMH Repositories
}

\author{
Noha Adly \\ Department of Computer and Systems Engineering \\ Faculty of Engineering, Alexandria University \\ Alexandria, 21544 Egypt \\ noha.adly@alex.edu.eg
}

\begin{abstract}
Metadata harvesting requires timely propagation of up-to-date information from thousands of Repositories over a wide area network. It is desirable to keep the data as fresh as possible while observing the overhead on the Harvester. An important dimension to be considered is that Repositories vary widely in their update patterns; they may experience different update rates at different times or unexpected changes to update patterns. In this paper, we define data Freshness metrics and propose an adaptive algorithm for the synchronization of the Harvester with the Repositories. The algorithm is based on meeting a desired level of Freshness while incurring the minimum overhead on the Harvester. We present a comparison between different policies for the synchronization within the framework devised. It is shown that the proposed policy outperform the other policies, especially for heterogeneous update patterns.
\end{abstract}

Keywords: Data Synchronization, Freshness Constraints, Distributed Objects, Harvesting, Digital Libraries, OAI-PMH.

\section{INTRODUCTION}

There is an exponential growth of online material and digital libraries play a key role in managing this information by structuring the content so that it is discovered easily and effectively. Many repositories use the Open Archives Initiative Protocol for Metadata Harvesting (OAI-PMH) [1] to expose metadata about their resources and contents. OAI-PMH is based on the standard technologies HTTP and XML as well as the Dublin Core metadata scheme. It is a set of six verbs or services that provides an open interface for metadata exchange and harvesting. Within OAI-PMH, a Data Provider is a Repository that exposes its structured metadata; and a Harvester, operated by a Service Provider, makes OAI-PMH service requests to harvest that metadata from repositories. Service Providers then provide value-added services, such as federated search $[2,3]$, on the harvested data extracted from the repositories. A general configuration of OAI-PMH is shown in Figure 1.

Selective harvesting allows harvesters to limit harvest requests to portions of the metadata available from a repository. The OAI-PMH supports selective harvesting and Harvesters are expected to exploit this property to limit the load placed on Repositories and Harvester while maintaining fresh data for services offered by the Service Provider. Selective harvesting is supported in OAI-PMH through timestamps, included as from argument in the ListRecords requests and expressed in seconds' granularity, which are used to harvest only those records that were created, deleted or modified within a specified range.

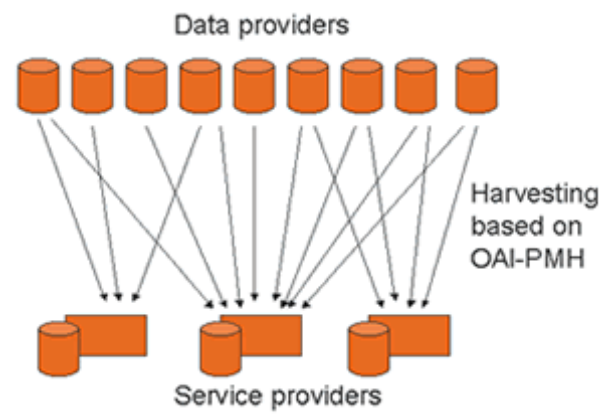

Figure 1: General configuration of OAI-PMH

The synchronization problem addresses how to keep the metadata records of the Repositories and Harvester consistent. Frequent harvesting results in the data at the Service Provider being up-to-date and consistent with the Data Provider. However, frequent harvesting results in a high overhead on both the Harvester and the Repositories, which renders the harvesting inefficient, especially if the Data Provider has not been updated during the harvest interval. On the other hand, without frequent harvesting, Service Providers may become inconsistent with Data Providers: not only can new records be missed, but deletions and modifications as well and hence mislead the results offered to the user by the Service Provider. The challenge is how to design a harvesting algorithm that strikes the balance between the Freshness of the data and the overhead incurred. 
A large number of repositories have been using OAI-PMH to expose their data, which are of different domains; ranging from scholarly publishing data such as E-print repositories $[4,5,6]$ or education material such as HEAL [7], multimedia resources, biomedical data [8], and archeological data [9]. These applications are likely to have a small but steady stream of daily or weekly updates. However, different applications started to arise that manage data of different nature. Recent initiatives [10] have proposed making usage data of scholarly information service, collected from web logs, available using OAI-PMH and focused on promoting its applications and creating value-added services on this data such as derivation of global measures of impact and the identification of global trends. Also, recently, there has been a growing interest in harvesting news, annotations [11], reviews of articles and RSS feeds. Those applications are likely to have a large number of updates and with high frequency.

Therefore, it is expected with current applications that Repositories would be heterogeneous in nature: different Repositories may have different update rate and a Repository may have different update rate in different time of the day. The update pattern of Repositories plays a major role in determining the balance between frequent harvesting, which guarantees Freshness of data at the expense of high overhead and infrequent harvesting which could result in stale data. Inconsistency or stale data, although could be acceptable in some applications, it would be undesirable for some applications e.g. news feeds, which are sensitive to data Freshness. Therefore there is a need for an adaptive policy that adjusts the harvesting according to the update patterns of the Repositories.

Other major potential users of OAI-PMH are search engines. Although, current commercial search engines make a limited use of OAI-PMH to index their data, a study [12] performed on 10 millions records of OAIPMH repositories revealed that Google, Yahoo! and MSN indexed only $60 \%, 44 \%$ and $7 \%$ respectively of these records. However, as interest in revealing site content to web crawlers in a structured manner has increased recently, it is expected that major search engines will support more of OAI-PMH in order to index more content. This will lead to a larger number of repositories registering and implementing OAI$\mathrm{PMH}$ to be able to share their contents. Hence, efficient harvesters are needed that will be able to pull data with dynamic behavior from a large number of Repositories.

This growing interest in variety of applications suggests that the environment will be much more dynamic than before, with a larger number of
Repositories to be harvested and with a variety of natures of Repositories, with different update patterns.

In this paper, we present an adaptive pull-based policy for harvesting data from a set of Repositories that aims to reduce the overhead on the Harvester, and consequently on the Repositories, while maintaining Freshness of data at a certain level. In order to ensure freshness of data without wasting resources, we provide a framework for measuring the Freshness of data at Harvester as well as the cost, which allowed devising an optimal algorithm for harvesting that is able to adapt to changes in the update patterns at Repositories. The algorithm presented is compliant with the OAI PMH protocol with minimal changes required at the Repository and the Harvester. It relies on piggybacking compact representation of the Repository workload on ListRecords response. It is shown that the proposed policy results in reduction in the cost on the Harvester compared to other policies while providing comparable level of freshness. The benefits have shown to be maximized for heterogeneous update patterns.

The structure of the paper is as follows. Section 2 discusses previous work in synchronization and measuring the freshness data. In section 3 , we present a framework for deriving measures for the Freshness of data and Cost on the Harvester that allows us to formulate the optimization problem and derive its solution. Section 4 presents the Optimal Adaptive Policy $O A P(\theta)$. In section 5, we provide a comparison between $O A P(\theta)$ and three other policies for harvesting. Finally, Section 6 concludes the paper.

\section{RELATED WORK}

Synchronization and Freshness problems arise in various contexts. In [13], several definitions of data Freshness and the metrics measuring them are introduced according to the applications where they are used; whether replications systems, federated databases, data warehousing, web portal, cashing systems, etc. They presented a taxonomy based upon the nature of the data, the type of the application and the synchronization policy used. Our work is driven by synchronizing Harvester with Repositories within OAI-PMH protocol.

Synchronization of large collection of objects, for example, web crawlers, has been addressed in [14, 15] where they have defined age and freshness metrics by modeling the average update frequency of individual elements of a database as well as the whole database. They analyzed different synchronization policies based on the frequency of synchronizing the local database, the frequency of synchronizing individual elements, the synchronization order and the synchronization 
points over time. However, their approach relies on discovering the update time of each individual web page, which is different than the incremental harvesting model of the OAI-PMH.

Labrinidis [16] considered freshness in the context of view materialization in caching dynamic web content. They studied selecting which views to materialize in order to maximize performance while keeping data freshness at acceptable level. A Quality of Data (QoD) metric was defined to evaluate how fresh the data served to the users is. They propose an algorithm which constantly monitors the QoD of served data and periodically adjusts the materialization plan by allocating more (or less) resources when there is a QoD deficit (or surplus). This study also focuses on individual web pages.

Driven by results showing that in web caching 30 $50 \%$ of cache hits result in unnecessary validations, which incur high latency, Bright et. al [17] presented two history-based policies, that establish their prediction on the repetitive nature of update history. They are an extension to the TTL (Time-To-Live) policy, where each object is assigned a TTL and a validation occurs for any cached object whose TTL has expired. The TTL value is usually estimated as a function of the time that an object was last modified. However, in [17] they are targeting an environment where updates patterns are non-homogenous, capturing updates in a timely manner is critical and some degree of staleness is unacceptable. Hence, they modeled update history of an object as a cyclic stochastic model that can be extended with bursts or deviations from the cyclic history. It has been shown that the history based policies outperform TTL either for cyclic update pattern or acyclic history that exhibits bursts. It should be noted that this approach is different than the OAIPMH synchronization because within web caching synchronization is done on the object level, that is, only when this particular object is accessed it is refreshed; while in OAI-PMH the synchronization is applied to all objects of the repository.

In [18], they studied the synchronization problem of the OAI-PMH. By examining the harvest logs of Arc[19], an OAI harvester for e-print services, they concluded that most repositories change at a steady rate, but the rates vary dramatically from site to site. They suggested four possibilities for adaptive policies for synchronization. The first is based on the Harvester estimating the update frequency by learning the harvest history and the second is based on the Repository notifying the Harvester of its update frequency as a response to an Identify request. Although both policies are OAI-PMH compliant, the details of the algorithms were not discussed. Also, relying on information sent through the Identify request is not adequate since this verb is used only for newly registering Repositories. Further, the metrics introduced for studying the synchronization were mainly used for formalization of some definitions and did not allow for quantification of the Freshness or the overhead that could help in evaluation of the proposed algorithms. The other two algorithms were based on either Repositories notifying the Harvester whenever content is changed or on a Push-based mechanism. Other than they have not been presented in details or evaluated, both proposals are not OAI-PMH compliant and would require major changes in the protocol.

\section{FRAMEWORK}

To study the synchronization problem, we present a framework that allows us to study and measure the metrics that affect the performance. One important measure is the quality of the data or, Freshness. The other metric which we take into consideration is the overheard, or the Cost, incurred on the Harvester.

\section{A. Freshness Measure}

When an element is updated at a Repository $R$, this element becomes stale with respect to the Harvester. The element remains stale until a harvest occurs where the value of the element at the Harvester is updated. Obviously, it is required that the data elements harvested be as fresh as possible, that is more up-todate. Let $\left\{R_{1}, R_{2}, \ldots . . R_{M}\right\}$ be $M$ Repositories to be harvested and $R_{i}=\left\{e_{1}, e_{2}, \ldots \ldots e_{N i}\right\}$ be Repository $i$ with $N_{i}$ elements.

An element in a Repository is considered fresh at time $t$ if it is up-to-date at time $t$ w.r.t. to the Harvester i.e. if its value at Repository is equivalent to its value at Harvester at time $t$. Otherwise the element is considered stale.

Definition 1: Freshness of element $e_{j}$ at time $t$.

$F\left(e_{j}, t\right)= \begin{cases}1 & \text { if } e_{j} \text { is up - to - date at time } t \\ 0 & \text { otherwise }\end{cases}$

The freshness of $R_{i}, F\left(R_{i}, t\right)$, is defined as the fraction of the $R_{i}$ that is up-to-date. $F\left(R_{i}, t\right)$ is a rational number between 0 and 1, with a value of one, if all elements of $R_{i}$ are up-to-date and would be zero if all elements are stale. Given that $R_{i}$ contains $N_{i}$ elements, $F\left(R_{i}, t\right)$ is the average of the freshness values of all elements that compose $R_{i}$.

$F\left(R_{i}, t\right)=\frac{1}{N_{i}} \sum_{j=1}^{N_{i}} F\left(e_{j}, t\right)$

Note that Freshness is hard to measure exactly in practice, since we need to instantaneously compare the data elements of the Repository to the Harvester. But it is possible to estimate Freshness given some 
information about how the elements of the Repository change. In order to measure Freshness, we observe the synchronization stream and the update stream for a certain observation period $T$. Assume that the average update rate of $R_{i}$ is $\lambda_{i}$ and that the Harvester performs $P_{i}$ pulls for each $R_{i}$ at regular intervals $I_{i}=T / P_{i}$. Figure 2 shows the evolution of updates with the horizontal axis representing the time and the vertical axis representing the number of stale items.

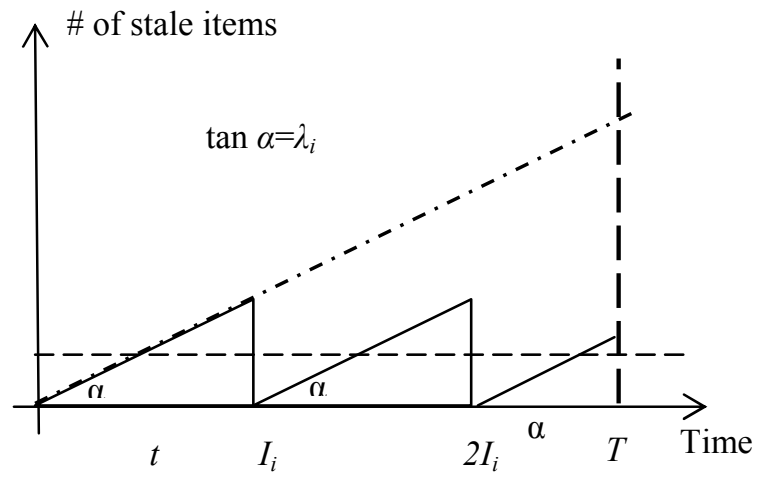

Figure 2: Evolution of updates at a Repository $R_{i}$

The synchronization stream during the observation period $T$ is viewed as a sequence of harvests requests made at time $I_{i}, 2 I_{i}, 3 I_{i}, \ldots$ Then the number of stale items at $R_{i}$ at time $t$ :

$$
S_{i}=\lambda_{i} * t \quad t=0,1,2, \ldots I_{i}
$$

Assume we synchronize at $t=0$ and $t=I_{i}$, then, from Figure 2 the average number of stale items:

$\overline{S_{i}}=\lambda_{i} * \frac{I_{i}}{2}$

The Freshness of a Repository $R_{i}: F_{R i}=1-\overline{S_{i}} / N_{i}$

$F_{R_{i}}=1-\frac{\lambda_{i} I_{i}}{2 N_{i}}=1-\frac{\lambda_{i} T}{2 N_{i} P_{i}}$

The Freshness of Harvester $H$ is defined as the average Freshness of all the Repositories it harvests.

$$
F_{H}=\frac{\sum_{j=1}^{M} N_{j} * F_{R_{j}}}{\sum_{i=1}^{M} N_{i}}=1-\frac{\sum_{j=1}^{M} \lambda_{j} I_{j}}{2 \sum_{i=1}^{M} N_{i}}
$$

\section{B. Cost Measure}

Another important measure that affects the performance is the overhead incurred on the Harvester. The Cost on the Harvester depends on the update rate $\lambda_{i}$ and the pull rate $P_{i}$ for each Repository it harvests. For each harvest, the Harvester extracts new records from $R_{i}$, which incurs a communication cost as well as a processing cost. This cost is paid even if there are no new records to harvest. Also, the Harvester extracts, processes and applies every new update to his local copy of the database. Let

$C_{U}=$ Cost incurred from extracting and processing a single update.

$C_{p}=$ Cost of initiation, negotiation and communication of a pull. Then,

$\left.\mathrm{C}_{\mathrm{H}}\right|_{\mathrm{Pi}}=$ Cost of Harvester for pulling $R_{i}$

$\left.C_{H}\right|_{P i}=T \lambda_{i} * C_{U}+P_{i} * C_{P}$

$C_{H}=C_{u} T \sum_{i=1}^{M} \lambda_{i}+C_{p} \sum_{i=1}^{M} P_{i}$

\section{Optimal Harvest Intervals}

This section will study how often a Harvester should pull each Repository, when it knows how often they change, in order to minimize the Cost while maintaining a certain level of Freshness. We formulate the problem as an optimization problem with the objective to determine the optimal harvest interval $I$.

Problem: Given $\lambda_{i}, N_{i}, F_{H}=\theta$, find $I_{i}$ which minimize the $\operatorname{cost} C_{H}$

$C_{H}(I)=C_{u} T \sum_{i=1}^{M} \lambda_{i}+C_{p} T \sum_{i=1}^{M} I_{i}^{-1}$

Given freshness $F_{H}=\theta$ or $\quad \theta=1-\frac{\sum_{j=1}^{M} \lambda_{j} I_{j}}{2 \sum_{j=1}^{M} N_{j}}$

We can solve the above constrained optimization problem using the method of Lagrange multipliers [20], where the constraint function is

$g(I)=\sum_{j=1}^{M} \lambda_{j} I_{j}-2(1-\theta) \sum_{j=1}^{M} N_{j}=0$

Define the Lagrangian $\Lambda$ as

$$
\begin{aligned}
\Lambda(I, \mu) & =C_{H}(I)+\mu \cdot g(I) \\
& =C_{u} T \sum_{i=1}^{M} \lambda_{i}+C_{p} T \sum_{i=1}^{M} I_{i}^{-1}+\mu\left[\sum_{j=1}^{M} \lambda_{j} I_{j}-2(1-\theta) \sum_{j=1}^{M} N_{j}\right]
\end{aligned}
$$

Solving for points $I$ where

$\nabla \Lambda(I, \mu)=\nabla C_{H}(I)+\mu . \nabla g(I)=0$

From the partial derivatives with respect to $I$, we can deduce

$$
I_{j}= \pm \frac{\sqrt{\lambda_{1}}}{\sqrt{\lambda_{j}}} I_{1} \quad \forall j=2 \rightarrow M
$$


Substituting into the partial derivate with respect to the Lagrange multiplier $\mu$

We get $I_{i}=\frac{2(1-\theta) \sum_{j=1}^{M} N_{j}}{\sqrt{\lambda_{i}} \sum_{j=1}^{M} \sqrt{\lambda_{j}}}$

and

$$
C_{H}(I)=C_{u} T \sum_{i=1}^{M} \lambda_{i}+C_{p} T \frac{\left[\sum_{i=1}^{M} \sqrt{\lambda_{i}}\right]^{2}}{2(1-\theta) \sum_{j=1}^{M} N_{j}}
$$

\section{Optimal AdAPtive Policy Algorithm}

Current harvesting algorithms are based on a fixed uniform harvest interval that is applied to all Repositories. Such algorithms will not work well in an environment where updates patterns change dynamically. The heterogeneous nature of Repositories workloads mandates that the harvesting algorithm, be adaptive in order to evolve under changing workload pattern.

In this section we propose an Optimal Adaptive Policy algorithm, $\operatorname{OAP}(\theta)$, a harvesting algorithm that is executed at the Harvester, where $\theta$ is a threshold specified by the Harvester. $\operatorname{OAP}(\theta)$ strives to maintain the overall Freshness above the specified threshold $\theta$ and also keeps the cost at the Harvester as low as possible.

$\operatorname{OAP}(\theta)$ is inherently adaptive. The algorithm relies on the Harvester collecting statistics from the Repositories concerning their workloads and computes the optimal intervals at which it pulls each Repository to achieve the level of Freshness desired $\theta$ while minimizing the cost incurred. Namely, the Harvester $H$ estimates $\lambda_{i}$ and $N_{i}$ for each $R_{i}$ and computes the optimal intervals from Equation (3). One main concern while devising $\operatorname{OAP}(\theta)$ is to be compliant with the OAI-PMH protocol with minimum or no changes introduced to the protocol.

The main OAI-PMH verb used by $\operatorname{OAP}(\theta)$ is the ListRecords verb, which is used to harvest records from a Repository based on a timestamp, where the from argument specifies the lower bound for the timestamp-based selective harvesting. OAI-PMH controls the return of large number of records through partitioning the records into batches and the use of a resumptionToken with each batch. This partitioning is accomplished as follows: a Repository replies to a ListRecords request with an incomplete list and a resumptionToken; in order to retrieve the next portion of the complete list, the next request from the
Harvester must use the value of that resumptionToken element as the value of the resumptionToken argument of the request. Finally, the response containing the incomplete list that completes the list must include an empty resumptionToken element. The complete list then consists of the concatenation of the incomplete lists from the sequence of requests.

$\operatorname{OAP}(\theta)$ will be using the resumptionToken as the mean to pass on the information needed from the Repository to the Harvester. It makes use of the fact that the resumptionToken is already incorporated into the protocol and has associated attributes that are useful to the implementation of $\operatorname{OAP}(\theta)$, without the need to change the operation of a verb or to introduce a new verb or to change the XML schema. Namely, completelistSize, an attribute associated with the resumptionToken, is an integer indicating the cardinality of the complete list to be sent; which basically represent the number of updates sent from the Repository during this harvest cycle. $\mathrm{OAP}(\theta)$ introduces a new attribute to the resumptionToken, which is totElements, indicating the total number of elements at the Repository at the time. These two values are passed with every response to a ListRecords request from the Repository to the Harvester. Therefore, $\operatorname{OAP}(\theta)$ suggests a minor change in the implementation of the ListRecords verb at the Repository side. More precisely, it suggests that the Repository includes a resumptionToken in every response to ListRecords, even if the need for partitioning does not arise. The resumptionToken will be empty if the whole set of updates are to be sent in one partition, and will have an identifier if the set of updates is partitioned. It should be noted that making resumptionToken mandatory for the Repository does not present an overhead since these attributes are sent with the whole list and not on the record level. Further, the values of the attributes totElements and completelistsize sent are already known to the Repository and do not need to be computed.

Harvester $H$ estimates the update rate of $R_{i}, \lambda_{i}$ from the number of updates it receives from $R_{i}$ in the current harvest. Let the number of updates $H$ receives from $R_{i}$ at Pull $j$ is $U_{i}^{R}$ and the total number of elements at $R_{i}$ received is $N_{i}^{R}$. The value of $U_{i}^{R}$ and $N_{i}^{R}$ represent the value of the completeListSize and totElements attributes of the resumptionToken transmitted from $R_{i}$ along with the response to the ListRecords request. $H$ can use the recursive prediction error method [21] to estimate the update rate in the near future. Namely, $\lambda_{i}^{H}=(1-g) \lambda_{i}{ }^{P}+g \lambda_{i}^{R}$, where

- $\lambda_{i}^{H}=$ new estimate of update rate of $R_{i}$ for the next period 
- $\lambda_{i}^{P}=$ old estimate for update rate in the last interval

- $\lambda_{i}^{R}=$ update rate for the current interval $=U_{i}^{R} / I_{i}$, where $I_{i}$ is the interval at which these updates occurred.

- $\mathrm{g}=$ gain factor, $0<\mathrm{g}<1$ suggested [21] to be set to 0.25

Although more sophisticated methods could be used by the Harvester for estimating the number of updates, it is believed that this heuristic is simple and incurs a small overhead. Basically, $H$ needs just to keep an array $\lambda_{i}^{P}$ of size $M$ that keeps the actual rates of updates at the current interval received from Repositories for use of the estimate of the number of updates for the next interval. So the storage space and the computational complexity are negligible. The Pseudo code for $\operatorname{OAP}(\theta)$ is as follows:

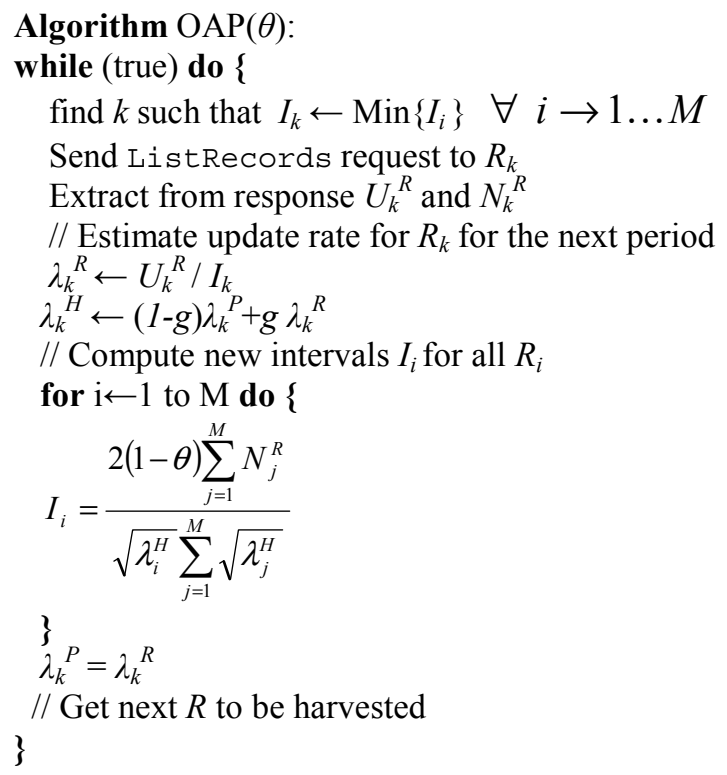

\section{COMPARISON BETWEEN DIFFERENT POLICIES}

In order to evaluate the potential benefits of the $\operatorname{OAP}(\theta)$, we provide a comparison between the $\operatorname{OAP}(\theta)$ and other policies for variant workloads. We represent the variation in the workload by considering four types of Repositories that exhibit different behaviors. Namely, we assume that a Repository can have a small number of elements $\left(N_{i}=1000\right)$ and others may have a large number of elements $\left(N_{i}=10,000\right)$. Further each Repository can have a small update rate $\left(\lambda_{i}=10 \% N_{i}\right)$ while another Repository can experience a large update rate $\left(\lambda_{\mathrm{i}}=50 \% N_{i}\right)$. This generates four types of Repositories simulating different behaviors as shown in Table 1.
Table 1: Four different types of workloads

\begin{tabular}{|l|l|l|l|l|}
\hline & $\mathrm{T} 1$ & $\mathrm{~T} 2$ & $\mathrm{~T} 3$ & $\mathrm{~T} 4$ \\
\hline$\lambda_{i}$ & 100 & 500 & 1000 & 5000 \\
\hline$N_{i}$ & 1000 & 1000 & 10000 & 10000 \\
\hline
\end{tabular}

It is assumed that the number of Repositories to be harvested $M=1000$ and the observation period is taken to be $T=1$ day. The cost of a harvest cycle is set to 50 units $\left(C_{P}=50\right)$, while the cost of extracting and processing a single update is set to $0.1\left(C_{U}=0.1\right)$.

\section{A. $O A P(\theta)$ vs. Uniform Adaptive Policy}

The objective of this experiment is to compare the Optimal Adaptive Policy $\operatorname{OAP}(\theta)$ with a Uniform Adaptive Policy (UAP). The UAP is set such that the Harvester pulls all the Repositories at a uniform (fixed) update interval $I_{U}$. This is compared to $\operatorname{OAP}(\theta)$ which sets a different pulling interval for each Repository according to the behavior of the Repository relative to the workload patterns of all other Repositories. In order to choose the Uniform Interval $I_{U}$, we assume that the Harvester is aware of the workload on each Repository, and hence computes $I_{U}$ as the Optimal Interval to achieve the required Freshness $\theta$ given that all Repositories are combined into a single site, which would result in the same Freshness as the $\operatorname{OAP}(\theta)$.

In this experiment, we assume that the Repositories are a mix of T1 and T4 workload; and we vary the percentage of Repositories that belong to T1 versus $\mathrm{T} 4$. That is, we evaluate a workload where $75 \%$ of the Repositories follow the pattern of type T1 and 25\% of them follow $\mathrm{T} 4$. Then we change this percentage till we reach $30 \%$ of Repositories of Type T1 and 70\% of type T4. We evaluate the overhead incurred on the Harvester for each policy for different Freshness thresholds $\theta$, as shown in Figure 3. We plot the ratio of the cost of UAP $C_{H}^{\prime}$ versus the cost of $\operatorname{OAP}(\theta), C_{H}$, $\left(C_{H}^{\prime} / C_{H}\right)$ for different mixes.

Results show that the gains of $\operatorname{OAP}(\theta)$ are higher when the mix of Repositories is inclined towards T1, with UAP suffering from increase in the cost ranging between $21 \%$ to $71 \%$ for different Freshness $\theta$. As the workload mix moves toward T4, the cost of UAP decreases, but is still higher than $\operatorname{OAP}(\theta)$, showing a degradation of $10 \%$ to $34 \%$ when the workload is evenly distributed between $\mathrm{T} 1$ and $\mathrm{T} 4$ and being $16 \%$ when the majority of Repositories are of T4. It is observed that as $\theta$ increases, the benefits of $\operatorname{OAP}(\theta)$ are more obvious where the degradation in UAP ranges between $71 \%$ to $16 \%$ for $\theta=0.98$ and $46 \%$ to $10 \%$ for $\theta=0.95$. This shows that the $\operatorname{OAP}(\theta)$ benefits are more dramatic for systems demanding high Freshness. Also, 
the $\operatorname{OAP}(\theta)$ is more adjustable to the variation of the workload mix than UAP.

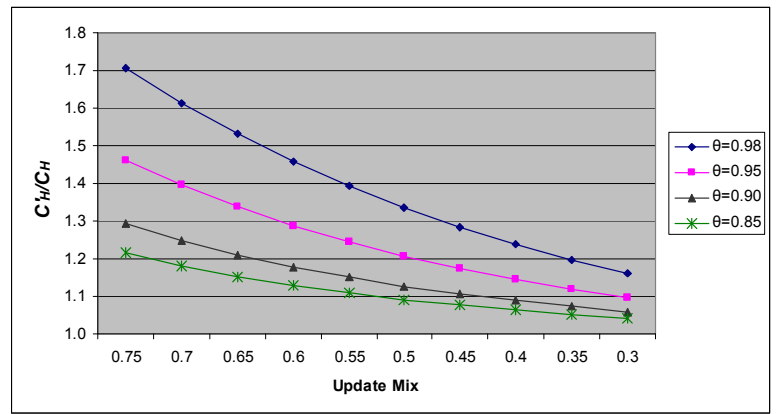

Figure 3: The Cost of UAP vs. OAP $(\theta)$ varying the workload mix

\section{B. $O A P(\theta)$ vs. Uniform Non-Adaptive Policy}

In this experiment, we compare $\operatorname{OAP}(\theta)$ with a Harvester that will apply a Uniform Policy as well; however, we assume that the Harvester is not aware of the actual mix between the Repositories he is about to harvest. Namely, he knows that the Repositories are a mix of $\mathrm{T} 1$ and $\mathrm{T} 4$, and that the mix would range between $80 \%$ to $60 \%$ of $\mathrm{T} 1$ versus $\mathrm{T} 4$. Hence he estimates that the mix would be $70 \%$ of T1 and $30 \%$ of T4 and it computes the uniform interval $I_{U}$ based on this estimate. We compare UNAP with $\operatorname{OAP}(\theta)$ in case the actual mix is ranging between the estimate $\pm 10 \%$. So we plot the variation in the mix between $80 \%$ and $60 \%$ and we evaluate the Freshness and Cost of both policies for different Freshness thresholds $\theta$, as shown in Figures 4 and 5.

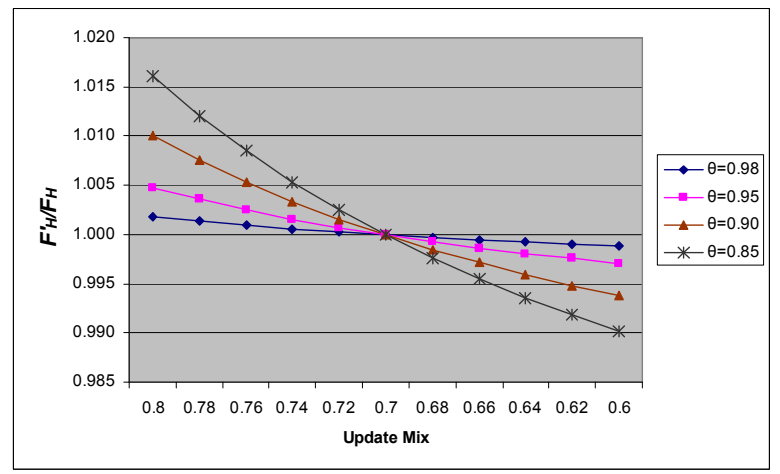

Figure 4: The Freshness of UNAP vs. OAP $(\theta)$

It is observed that when the actual mix is of $\mathrm{T} 1=70 \%$, which matches the estimates of UNAP, both policies have the same Freshness, while UNAP has a higher overhead in the cost ranging between $18 \%$ to $60 \%$ for different $\theta$. When the actual mix moves towards T1, UNAP experiences degradation in the cost ranging between $30 \%$ to $95 \%$ while the Freshness of UNAP is superior to that of $\operatorname{OAP}(\theta)$ by a range of
$0.1 \%$ to $0.001 \%$. When the actual mix moves towards T4, the cost of UNAP decreases, but still is higher than $\operatorname{OAP}(\theta)$ by a range of $11 \%$ to $4 \%$. This comes at the expense of the Freshness which decreases by a range of $0.1 \%$ to $0.001 \%$.

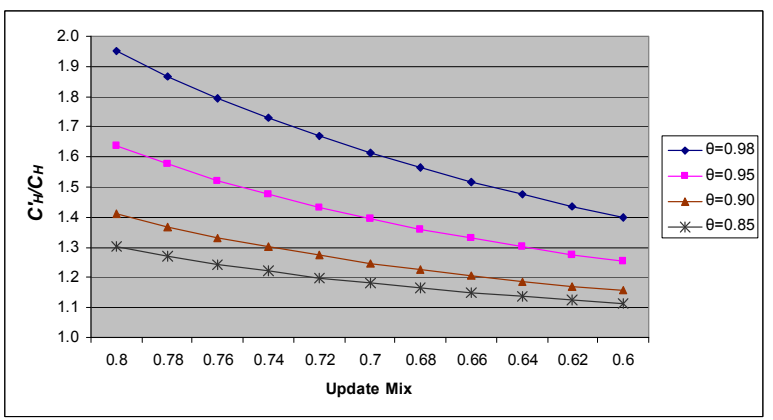

Figure 5: The Cost of UNAP vs. OAP $(\theta)$

\section{C. $O A P(\theta)$ vs. Individual Optimal Adaptive Policy}

In this experiment we compare $\operatorname{OAP}(\theta)$ with a different Adaptive Policy IOAP. In IOAP, the Harvester chooses the Optimal Interval for each $R_{i}$, based on the overall Freshness desired and the workload on this particular $R_{i}$, independently, rather than relative to the workload on all Repositories. This policy is simpler, since the Harvester would not need to recompute the optimal intervals each time he receives an update in the workload of one of the Repositories, as is the case in the $\operatorname{OAP}(\theta)$. IOAP results in same Freshness as $\operatorname{OAP}(\theta)$ but different costs, so we compare the cost of both policies for different Freshness thresholds $\theta$.

Figure 6 shows the ratio of the cost of IOAP $C_{H}^{\prime}$ to the cost of $\operatorname{OAP}(\theta) C_{H}$ while varying $\theta$ from 0.5 to 0.95. Results are shown for five cases representing different workload mixes of T1, T2, T3 and T4. In the first four cases, case $i$ represents a mix of a majority (70\%) of Repositories following type $T_{i}$, while $30 \%$ of the Repositories are uniformly distributed among the three other types. The fifth case represents a uniform mix of the Repositories between the different four types.

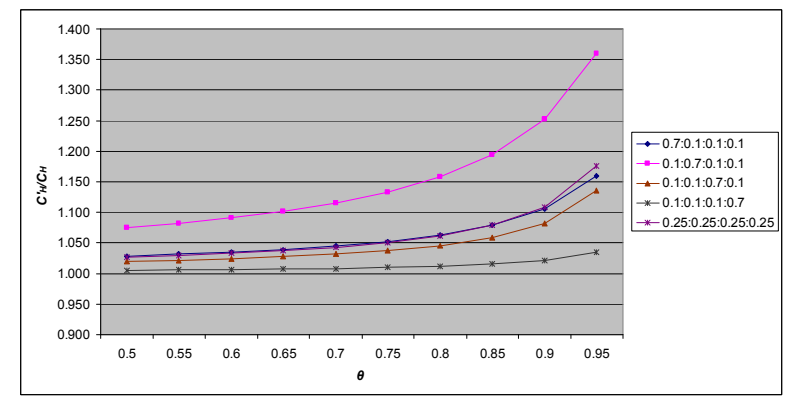

Figure 6: The Cost of IOAP vs. OAP $(\theta)$ varying $\theta$ 
Results shown in Figure 6 show that when the Repositories are evenly distributed between the different types of workloads, the IOAP incurs a higher cost ranging from $3 \%$ to $18 \%$, with higher overhead for higher $\theta$. When majority of Repositories are of type T2, IOAP behaves very badly with degradation reaching $36 \%$. A majority mix of $\mathrm{T} 1$ or $\mathrm{T} 3$ show similar behavior as the even mix while T4 is the least sensitive.

The above experiments show that $\operatorname{OAP}(\theta)$ captures the different mixes of workload and adjusts itself such that it provides major improvement over other policies in the cost, given a required threshold of Freshness.

It is expected that the performance of $\operatorname{OAP}(\theta)$ is dependent on the estimates of $\lambda_{i}$. However, we can show that $\operatorname{OAP}(\theta)$ is insensitive to the variations of $\lambda_{i}$ as long as the actual $\lambda_{i}$ deviates from the estimate of $\lambda_{i}$ by a value of $\pm \delta \lambda_{i}$. That is in the variations of the actual arrival rate, the amount of $+\delta \lambda_{i}$ is equal to $-\delta \lambda i$. For the cost, $C_{H}$, Equation (2) shows that the second term is independent of the actual $\lambda_{i}$. The first term, $C_{u} \sum_{i=1}^{M} \lambda_{i}$ is a summation of actual $\lambda_{i}$. Since $\left|+\delta \lambda_{i}\right|=\left|-\delta \lambda_{i}\right|$, then the total cost incurred by the variation of actual $\lambda_{i}$ would be equal to 0. Similarly, for the Freshness, from Equation (3) it is clear that $I j$ are independent of the actual arrival rate since the Harvester computes $I j$ based on the estimates of $\lambda_{i}$, not the actual. The term $\sum_{j=1}^{M} \lambda_{j} I_{j}$, which depends on actual $\lambda_{i}$ would lead to $\lambda_{i} \pm \delta \lambda_{i}$ canceling each other.

\section{CONCLUSIONS}

In this paper, we introduced an adaptive policy for harvesting OAI-PMH Repositories that experience different workload patterns. A framework is provided within which the Harvester can decide on the pulling frequency based on a desired level of Freshness while incurring a minimum overhead. It has been shown that the adaptive policy reduces the overhead on the Harvester, and hence on the Repositories, compared to other adaptive or uniform pull-based policies while offering comparable level of Freshness. This is especially obvious when the Repositories are heterogeneous and experience different update patterns.

\section{REFERENCES}

[1] C. Lagoze, H. Van de Sompel, M. Nelson and S. Warner. The Open Archives Initiative Protocol for Metadata Harvesting, version 2.0. http://www.openarchives.org/OAI/openarchivesprotocol.html
[2] K. Maly, M. Zubair and L. Xuemei, A High Performance Implementation of an OAI-Based Federation Service, In Proceedings of the $11^{\text {th }}$ International Conference on Parallel and Distributed Systems (ICPADS'05), 2005

[3] K. Maly, M. Zubair, V. Chilukamarri and P. Kothari, GRID Based Federated Digital Library, In ACM Proc of the $2^{\text {nd }}$ Conf. on Computing Frontiers, Ischia, Italy, May 2005

[4] arXiv. http://arxiv.org

[5] D-lib Magazine. http://www.dlib.org/

[6] D. Contessa and J. Moreira de Oliveira, An OAI Data Provider for JEMS, In Proceedings of the 2006 ACM Symposium on Document Engineering, Netherlands, 2006

[7] S. Mclntyre, S. Dennis, S. Uijtdehaage and C. Candler, A Digital Library for Health Sciences Educators: The Health Education Assets Library (HEAL), in Proc. of $4^{\text {th }}$ ACM/IEEE Joint Conference on Digital Libraries, June 2004

[8] Y. Fu and J. Mostafa, Integration of Biomedical Text and Sequence OAI Repositories, in Proc. of $4^{\text {th }}$ ACM/IEEE Joint Conference on Digital Libraries, June 2004

[9] N. Vemuri, R. Shen, S. Tupe, W. Fan and E. Fox, ETANA-ADD: An Interactive Tool for Integrating Archeological DL Collections, in Proc. of $6^{\text {th }} A C M / I E E E$ Joint Conf. on Digital Libraries, North Carolina, June 2006

[10] J. Bollen and H. Herbert Van de Sompel, An Architecture for the Aggregation and Analysis of Scholarly Usage Data, in Proc. of $6^{\text {th }}$ ACM/IEEE Joint Conference on Digital Libraries, North Carolina, pp 298-307, June 2006

[11] J. Hunter, I. Khan and A. Gerber, HarvANA Harvesting Community Tags to Enrich Collection Metadata, in Proc. of $8^{\text {th }}$ ACM/IEEE Joint Conference on Digital Libraries, Pittsburg, Pennsylvania, 2008

[12] F. McCown, M. Nelson, M. Zubair and X. Liu, Search Engine Coverage of the OAI-PMH Corpus, IEEE Internet Computing, April 2006

[13] M. Bouzeghoub and V. Peralta, A Framework for Analysis of Data Freshness, In Proceedings of the international Workshop on Information Quality in Information Systems ACM IQIS, Paris, 2004

[14] J. Cho and H. Garcia Molina, Synchronizing a Database to Improve Freshness, In Proc. of the 2000 ACM SIGMOD Intl. Conf. on Management of Data, Dallas, TX, May 2000

[15] J. Cho and H. Garcia Molina, Estimating Frequency of Change, ACM Transactions on Internet Technology, 3, pp 256-290, 2003

[16] A. Labrinidis and N. Roussopoulos, Exploring the Tradeoff between Performance and Data Freshness in Database-driven Web Servers, The VLDB Journal, 13, 3, pp 240-255, 2004

[17] L. Bright, A. Gal and L. Raschid, Adaptive Pull-Based Policies for Wide Area Data Delivery, ACM Transactions on Database Systems, Vol 31, No. 2, pp 631-671, June 2006

[18] X. Liu, K. Maly, M. Zubair and M. Neslon, Repository Synchronization in the OAI Framework, in Proc. of $3^{\text {rd }}$ ACM/IEEE Joint Conference on Digital Libraries, June 2003

[19] X. Liu, K. Maly, M. Zubair and M. Nelson, Arc - An OAI Service Provider for Cross-Archive Searching, in Proc. of $1^{\text {st }}$ ACM/IEEE Joint Conf. on Digital Libraries, June 2001

[20] G. B. Thomas, Jr. Calculus and analytic geometry. Addison-Wesley, $4^{\text {th }}$ edition, 1969.

[21] V. Jacobson, Congestion Avoidance and Control, in Proc. of ACM SIGCOMM, Stanford, CA, pp 314-329, 1988 